\title{
Deterministic removal strategy for machine vision assisted scanning micro electrochemical flow cell
}

\author{
Cheng Guo ${ }^{\mathrm{a}, \mathrm{b}}$, Jun Qian ${ }^{\mathrm{a}, \mathrm{b}}$, Dominiek Reynaerts ${ }^{\mathrm{a}, \mathrm{b}, *}$ \\ a Department of Mechanical Engineering, KU Leuven, 3001 Heverlee, Belgium \\ ${ }^{\mathrm{b}}$ Flanders Make, Belgium
}

\section{A R T I C L E I N F O}

\section{Keywords:}

Electrochemical machining (ECM)

Scanning micro electrochemical flow cell

(SMEFC)

Deterministic removal

Machine vision

Two-phase flow

\begin{abstract}
A B S T R A C T
Small cavities on a sliding surface can improve lubrication performance, which has been verified by many researchers. Electrochemical machining (ECM) is an effective way to fabricate this kind of small cavities on a large scale. When the diameter and the removal volume for the cavities are specified, it is still required to efficiently determine the appropriate machining parameters. This paper presents a machine vision based control system for an ECM variant: the scanning micro electrochemical flow cell (SMEFC). The aim of this system is to control the diameter of the cavity in real-time. With the assistance of machine vision, fast acquisition of the machining parameters for the specified diameter and the specified removal volume is possible. The system configuration is first explained in detail, including hardware and software configuration and the image processing algorithms. The latter are based on the Shi-Tomasi corner detector and are used for feedback control, stability and symmetry evaluation of the electrolyte droplet. For convenience, all of these functions have been integrated into a self-developed unified G-code interface. Furthermore, the theoretical explanation for controlling the machining process by vacuum gap (VG) tuning has been investigated through a two-phase flow simulation model, which revealed how the VG influences the shear rate and the pressure difference near the meniscus. Finally, a case study shows how to use the proposed strategy to get suitable machining parameters for a cavity with a diameter of $900 \mu \mathrm{m}$ and a target removal volume of $0.03 \mathrm{~mm}^{3}$. This demonstrates the availability of a deterministic removal strategy.
\end{abstract}

\section{Introduction}

\subsection{Deterministic removal for cavity machining}

It is widely known [1-3] that arrays of small cavities on a sliding surface have the potential to reduce friction in sliding pairs, because these cavities provide lubrication and can serve as micro hydrodynamic bearings during sliding. More specifically, the cavity area ratio, cavity depth and cavity diameter have significant influences on the friction performance [4]. In other words, both the cavity area and the cavity volume are important, since the cavity area influences the carrying capacity of the sliding surface and the cavity volume influences the space for the lubricating media. Therefore, it is very important to have a deterministic control of the geometry of the cavities or asperities [5]. In this paper, "deterministic removal" is therefore defined as the case, in which both the specified cavity diameter and the specified cavity volume can be controlled (Fig. 1) simultaneously. When applying a cutting process, it is relatively easy to achieve deterministic removal by optimizing tool design and path planning. Also for laser micromachining advanced modeling and learning techniques have been applied to produce deterministic geometries [6]. On the other hand it is for electro-chemical machining (ECM) difficult to quickly obtain suitable parameters for deterministic machining due to the fact that electrochemical dissolution acts in all directions.

In the ECM domain, jet-ECM and through-mask ECM [7,8], as shown in Fig. 2 (a) and (b), are two frequently applied techniques, enabling the generation of the small cavities without edge lips or burs, and they have been successfully applied in tribology performance improvement $[9,10]$. As for these techniques, the diameter and the removal volume of the cavities change simultaneously during the electrochemical dissolution. For jet-ECM, it is a common technique to tune the machining current densities and machining time to deterministically achieve specified dimensions of cavities [11-13]. Similarly, the dimensions of cavities fabricated by through-mask ECM methods $[14,15]$ are controlled by the applied voltage (current density), machining time and even the mask wall angle [15]. Simulation models also contribute to the selection of the suitable machining parameters for deterministic removal in jet-ECM and through-mask ECM. Nevertheless,

\footnotetext{
* Corresponding author at: Department of Mechanical Engineering, KU Leuven, 3001 Heverlee, Belgium.

E-mail addresses: cheng.guo@kuleuven.be, guochenghit@gmail.com (C. Guo), jun.qian@kuleuven.be (J. Qian), dominiek.reynaerts@kuleuven.be (D. Reynaerts).
} 


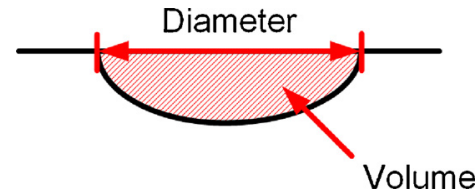

Fig. 1. Schematic of a cavity, where simultaneous control of diameter and volume is required to obtain a deterministic geometry. (a)

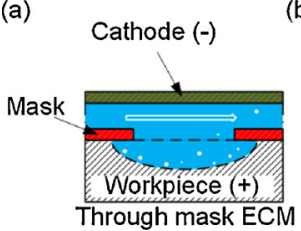

(b) Nozzle (-)

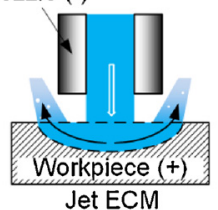

(c)

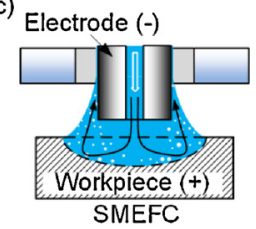

Fig. 2. Schematic of (a) through-mask ECM, (b) jet-ECM and (c) SMEFC.

the searching process for suitable parameters is time-consuming, since a lot of experiments are needed, even in the case of a well-planned design-of-experiments approach.

\subsection{Scanning micro electrochemical flow cell}

The scanning Micro Electrochemical Flow Cell (SMEFC) (Fig. 2 (c)) principle, as a kind of electrochemical machining method, has been already investigated in the past work of the authors: mesoscale cavities [16] and channels [17] were fabricated by this technique. The principle and schematic of the SMEFC setup are illustrated in Fig. 3. The difference with conventional ECM milling and drilling is the addition of a suction head encircling the hollow electrode. The electrolyte is pumped into the central hole of the electrode as usual. In this case however, the electrolyte will not spread on the workpiece surface, but is sucked back into the suction head through the small gap ring between the suction head and tool electrode. This is realized using fast flowing air around the electrode tip and using the negative pressure generated by a Venturi tube. This method avoids electrolyte splashing and maintains the electrolyte refreshment. As a result, the electrochemical dissolution only happens in the area occupied by the electrolyte and a small cavity will be generated. Because of the specific electrolyte circulation, there is no need for the workpiece to be immersed in the electrolyte, enabling SMEFC to be a portable technique. The cavity ratio can be adjusted by changing the vacuum gap (VG) [16], which is the distance between the bottom surface of the suction head and the workpiece top surface, as indicated in Fig. 3. The fundamental principles governing the cavity ratio will be explained in this work.

Fig. 3(a) also shows the microscope and light source, by which the visual information on the electrolyte droplet in SMEFC can be obtained. This machine vision system makes on-line control of the cavity diameter possible. For jet-ECM and through-mask ECM, due to the invisibility of the flow field in the machining region, this kind of machine vision assisted method is very difficult to realize. When the cavity diameter can be monitored in SMEFC by visual information, it will be easier to control the machining parameters for a deterministic removal, where both the removal volume and diameter of the cavity are specified. This will be further explained in the following paragraphs.

\subsection{Machine vision applications in manufacturing}

Machine vision, as a powerful monitoring and sensing method, has already substantially improved machine tools' controllability and intelligence. Yan et al. [18] applied a machine vision system in microEDM to achieve a new electrode compensation method, which reduced $40 \%$ of the machining time compared to the uniform wear method. Fernández-Robles et al. [19] developed a machine vision system to automatically detect broken inserts in edge profile milling heads, which is suitable to be integrated into an on-line machining system. Wang et al. [20] introduced a stereo vision system to automate hybrid manufacturing process planning. Liu et al. [21] utilized a similar system to detect the defect area of metallic components, accelerating the process for automatically targeting the boundary of the defect area. AbdulAmeer et al. [22] proposed a vision-based sensing utility, consisting of two cameras, to enhance the CNC milling performance. The vision information of which improved the surface roughness by dynamically changing the machining parameters. Sitthi-Amorn et al. [23] applied an integrated machine vision system in a self-developed multi-material 3D printing platform. This machine vision system can achieve self-calibration of printheads, 3D scanning, and a closed-feedback loop to enable print corrections, which simplifies the overall platform design. Supriadi et al. [24] put forward a vision-based fuzzy control method for dieless tube drawing to enhance the dimensional accuracy. Hung et al. [25] proposed using machine vision techniques to assist the resharpening process of micro-drills, which make it possible to automatically obtain all required grinding parameters. In addition, many successful applications have been achieved in the welding domain [26-28].

In the sinking ECM process, cameras usually function as an in-situ offline measurement [29-31]. Paczkowski et al. [32] used a Chargecoupled Device (CCD) camera to obtain geometric elements such as inter-electrode gap, working as a basis for the tool electrode position

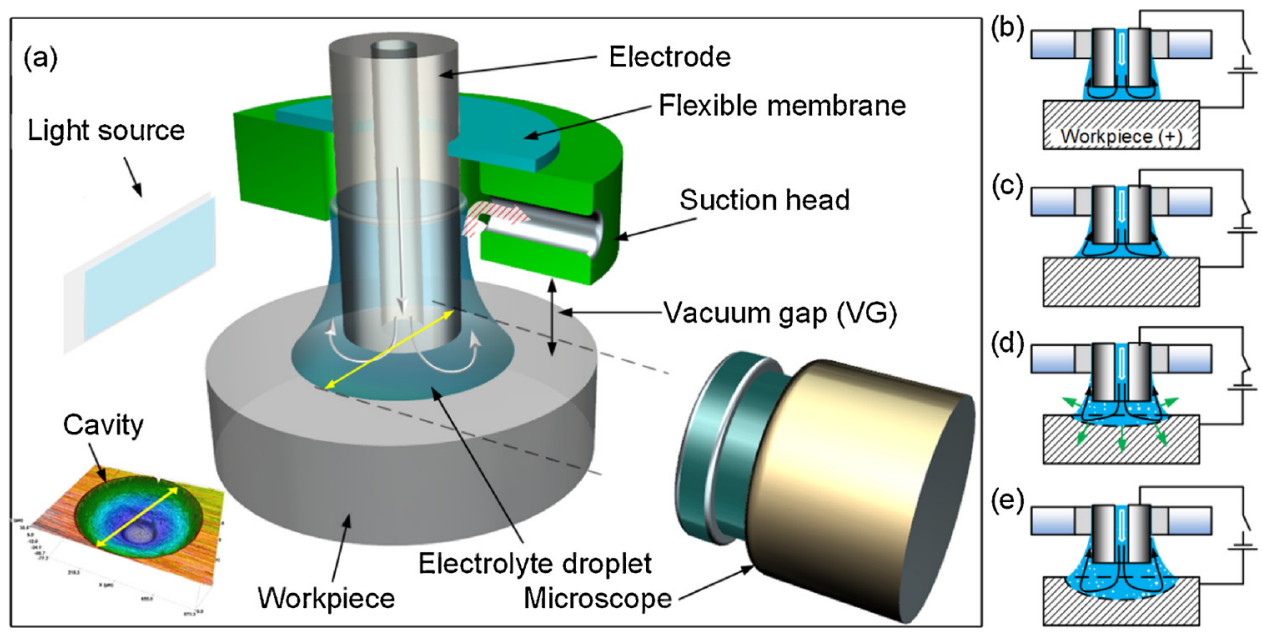

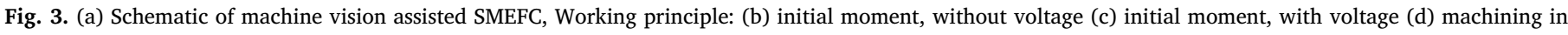
progress (e) final state, without voltage. 


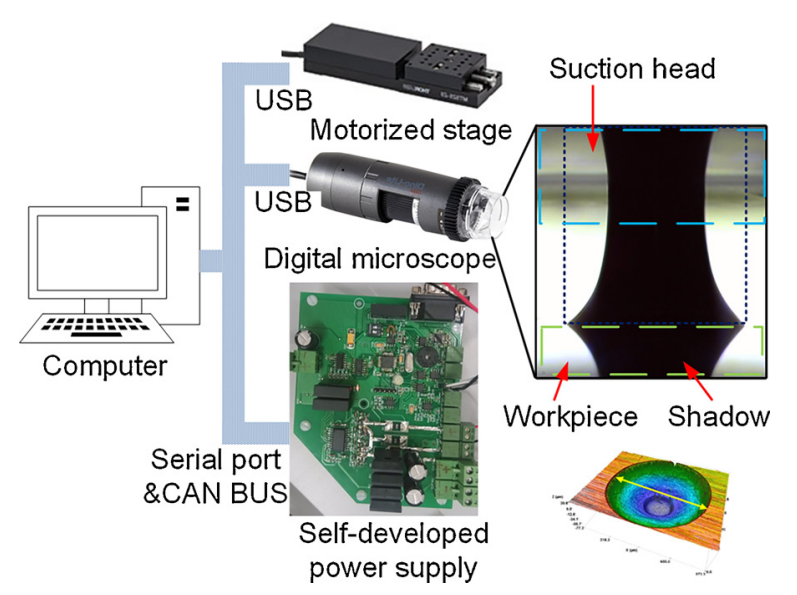

Fig. 4. Schematic of the control system.

correction. To the authors' knowledge, there is still no literature reporting the application of machine vision for the on-line controlling the ECM processes.

In this paper, the construction of a machine vision assisted SMEFC is discussed in detail ranging from hardware to software. The algorithms for feedback control of the diameter of the cavity and stability evaluation of the electrolyte droplet are explained, including the parameter tuning scheme and governing principles. Next, the experiments to validate the performance of the machine vision based control system will be discussed. The results indicate that this control system can automate the cavity diameter control, stability evaluation and problem detection, which pave the way for the application of SMEFC in practical manufacturing chains. The machine vision assisted system can speed up the searching process for machining parameters, which achieve deterministic removal.

\section{Machine vision assisted SMEFC}

\subsection{Hardware and software}

The different control system components are shown on Fig. 4, consisting of a self-developed ECM power supply, a digital microscope, a motorized stage and a PC, on which the customized software runs.

The key specification of the PC in the control system are listed in Table 1.

For the software, an open source C ++ library Qt was utilized to act as the GUI framework. The OpenCV 3.0 library was used to achieve real-time computer vision based on its optimized programming functions. Another recent successful application of the OpenCV library is an automated droplet measurement (ADM) constructed by Chong, Z.Z. et al. [33]. In addition, a customized G-code interpreter was developed with the help of Lex and Yacc, two GNU tools, to unify the control interface. The integrated development environment (IDE) was Visual Studio 2015 (VS 2015) Community edition.

The microscope in the machine vision system was a Dino-Lite Edge AM4115ZT microscope. The key parameters of this digital microscope are listed in Table 2. The measurement resolution of the bare microscope under a magnification of $220 \times$ is $1.43 \mu \mathrm{m}$, which is enough for

Table 1

Configure of the computer.

\begin{tabular}{ll}
\hline Item & Description \\
\hline Operating system & 64-bit Windows 7 Professional \\
Processor & Intel(R) Core(TM)2 Duo CPU E8400 @ 3.00GHz \\
Memory (RAM) & $8.00 \mathrm{~GB}$ \\
Manufacturer & Dell \\
\hline
\end{tabular}

Table 2

Key parameters of the microscope.

\begin{tabular}{ll}
\hline Item & Description \\
\hline Magnification range & $20 \times-220 \times$ \\
Resolution & 1.3 Megapixels $(1280 \times 1024$ pixels $)$ \\
Frame rate (max) & $30 \mathrm{FPS}$ \\
LED type & White \\
Dimensions & $10.5 \mathrm{~cm}(\mathrm{~L}) \times 3.2 \mathrm{~cm}(\mathrm{D})$ \\
Depth of field & $0.1 \mathrm{~mm}$ \\
Working distance $(220 \times)$ & $11.9 \mathrm{~mm}$ \\
View field $(220 \times)$ & Around $1.8 \mathrm{~mm} \times 1.4 \mathrm{~mm}$ \\
Resolution $(220 \times)$ & $1.43 \mu \mathrm{m} /$ pixel \\
\hline
\end{tabular}

the measurement. Back lighting is utilized to create dark silhouettes against a bright background, as shown in Fig. 4. The measurement uncertainty for machine vision of the electrolyte droplet is more complicated, as it is subject to the lighting condition, the pixel dimension, the focused accuracy and even the software algorithm. As a reference measurement is also not available the measurement uncertainty for the droplet size could not be determined. Another solution for this problem is devised in Section 2.3.

The ECM power supply supports current-constant mode, voltageconstant mode and various pulse modes. The output ripples for the voltage-constant mode are less than $20 \mathrm{mV}$ and its response time is less than $50 \mathrm{~ms}$. The motorized stage is an MTS25-Z8 of THORLABS with the travel range of $25 \mathrm{~mm}$ and the maximum velocity of $2.4 \mathrm{~mm} / \mathrm{s}$. The smallest achievable incremental movement is $0.05 \mu \mathrm{m}$ and the bidirectional repeatability is $1.6 \mu \mathrm{m}$.

The pump used in the previous work [16] was a solenoid metering pump, the achievable minimum stable flow rate of which was $3.6 \mathrm{~mL} /$ min at a pulsation frequency of $3 \mathrm{~Hz}$. In this work, a peristaltic pump with 8 rollers is utilized instead of the old solenoid metering pump, because the new pump can achieve a higher resolution, a lower stable flow rate and a higher pulsation frequency. The achievable minimum and maximum stable flow rate of the peristaltic pump are respectively $0.44 \mathrm{~mL} / \mathrm{min}$ and $3.186 \mathrm{~mL} / \mathrm{min}$. For example, when the flow rate of this pump is set to $3.186 \mathrm{~mL} / \mathrm{s}$, the pulsation frequency is $12 \mathrm{~Hz}$, which means at the same level of flow rate, the peristaltic pump provides more stable (i.e. with less pressure variation) flow. This stable flow contributes to stabilizing the meniscus of the electrolyte droplet and reducing fluctuations during the machining process.

\subsection{Preprocessing}

After tuning well the exposure time, the function of automatic exposure adjustment of the microscope is disabled. The microscope is focused on the lateral boundary of the electrode before the electrolyte supply starts as shown in Fig. 5.

In order to reduce the calculation burden, the region of interest (ROI) containing the contact area should be selected from the original frame, as demonstrated in Fig. 6. Since it is difficult to have the camera

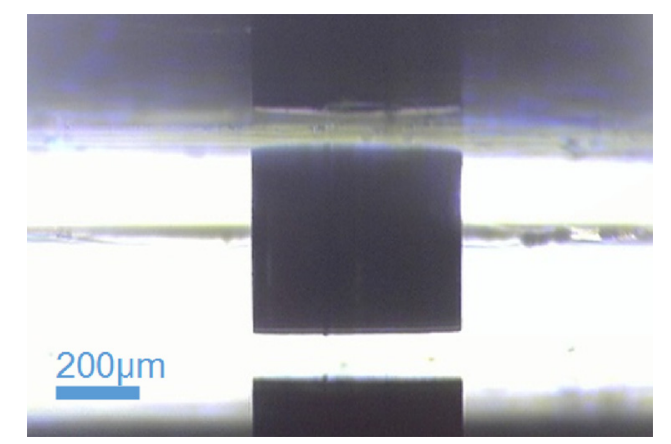

Fig. 5. Focusing on the side boundary of the working electrode. 


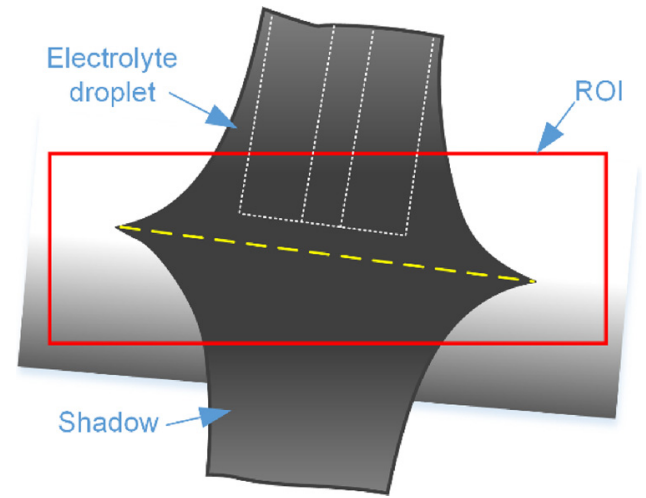

Fig. 6. Selection of the ROI selection.

completely (horizontally) aligned to the electrolyte droplet, the oblique is also considered as shown in Fig. 6. For general applications, a mark point will help to automatically locate the target, but setting up a mark point is in this case inconvenient. All the following processing algorithms are based on this ROI selection method.

\subsection{Feedback control of the cavity diameter}

In order to trace the cavity diameter, some feature should be detected to determine the endpoints of the electrolyte droplet diameter (yellow line in Fig. 6). As shown in Figs. 4 and 6, there is during the machining process a reflection of the electrolyte droplet on the ground surface of the workpiece, which generates two sharp corners at the endpoints. The two corners can be located through a series of image processing methods.

One method is based on the binarization of the ROI, as illustrated in Fig. 7(a), which originates from the post-processing method used in the past work [16]. Through this binarization processing, the electrolyte droplet and its shadow can be distinguished from the surrounding area. The output binary image has 255 for all pixels in the input image with

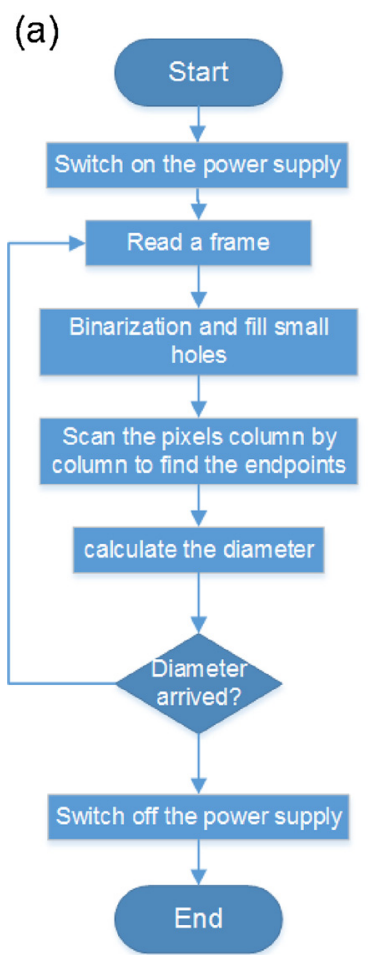

(b)

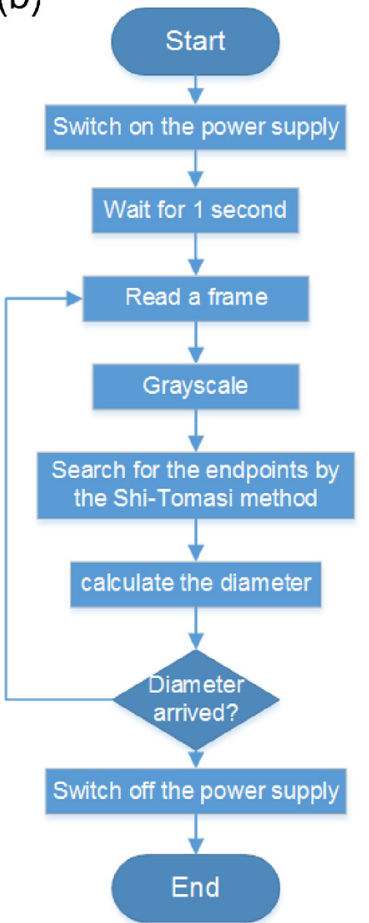

Fig. 7. Cavity diameter feedback control flow charts (a) Binarization method (b) Shi-Tomasi method. luminance greater than a thresholding in the range of [0,255], and 0 for all other pixels. Next, through scanning of the pixels column by column, the two endpoints of the contact line can be detected and finally, the length can be calculated. The scanning direction can be set to either from the middle outward to both sides or from both sides inward to the middle. After the binarization process, a function for filling image regions and holes can be applied.

The premise of this method is that the background of the droplet and its shadow should be clean enough to be distinguished. So this method is sensitive to the background quality and the lighting condition. If there are some previously machined cavities or big particles near the selected ROI, the detecting process tends to be influenced.

For the purpose of adaptability to different lighting and workpiece surface conditions, the corner detector, Shi-Tomasi method [34], has been finally utilized in this work. The Shi-Tomasi method is based on a grayscale image, so more visual information can be used. Although it is well known that corner detectors are still not stable and usually need an extra redundancy algorithm to fix errors, the Shi-Tomasi method quite matches the requirements of this research. The size of an average block for computing a derivative covariation matrix over each pixel neighborhood is set to 9. As shown in Fig. 8, there are 6 corners in the ROI, including 2 sharp corners (Corner 1 and 4) formed by the droplet and its shadow, which are the target corners. Compared with other corners, the 2 sharp corners are "stronger" corners in terms of the Shi-Tomasi method. By sorting the detected corners based on quality in a descending order, the two sharp corners can be easily picked out. As a result, the diameter of the machining area can be calculated based on these 2 corners. The flowchart of the whole processing procedure is illustrated in Fig. 7(b). The aim of the 1-second wait is to avoid a misjudgment due to the drastic changes in the droplet morphology at the initial droplet formation, during which the droplet changes from Fig. 3 (b) to Fig. 3 (c).

A self-developed G-code interface supports the branch and loop instructions to facilitate programming. Table 3 lists a set of G-code commands, which follow the flow chart in Fig. 7 (b). The function of this set of G-code commands is to continuously generate 8 cavities with a diameter of $900 \mu \mathrm{m}$. The inner and outer diameter of the hollow electrode are $180 \mu \mathrm{m}$ and $500 \mu \mathrm{m}$ respectively. The machining current is $300 \mathrm{~mA}$. The electrolyte is $\mathrm{NaNO}_{3}$ aqueous with a concentration of $250 \mathrm{~g} / \mathrm{L}$. The workpiece material is stainless steel (Uddeholm Stavax ESR AISI420). The workpiece has been treated by a surface grinder and cleaned by ethanol.

The cavities generated by the G-code of Table 3 are shown in Fig. 9. The overview of these cavities was taken by KEYENCE digital microscope. The first line of cavities (\#1 to \#8) were processed under the a VG setting of $350 \mu \mathrm{m}$ and the second line of the cavities (\#9 to \#16) were processed under a VG setting of $300 \mu \mathrm{m}$. The 3D image of the cavities were captured by SENSOFAR $S$ lynx surface profiler. The corresponding machining time $(\mathrm{T})$, the diameter (D) and the removal

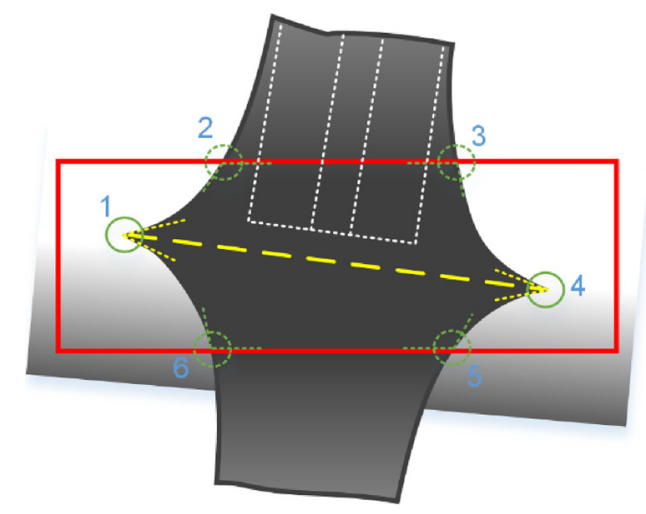

Fig. 8. Schematic of corners in the ROI. 
Table 3

G-code for diameter control.

\begin{tabular}{ll}
\hline G-code & Comment \\
\hline G91; & Relative mode \\
SEL $1 ;$ & Select the parameters of Group 1 \\
V1 $=0 ;$ & Empty the counter of the cavities to be machined \\
V2 = 900; & Set the target diameter \\
LTESTA: & Label \\
POWERON; & Switch on the power supply \\
G04 1000; & Wait for 1000 ms \\
M02 1; & Start image processing \\
TIMER1 1; & Initiate and start Timer 1 \\
LTESTB: & Label \\
G04 30; & Wait for 30 ms \\
IF DIA < V2 THEN GOTO & Judgment; DIA is a built-in parameter indicating \\
LTESTB; & the real-time diameter of the cavity. \\
POWEROFF; & Switch off the power supply \\
TIMER1 0; & Stop Timer 1 \\
PRINT T1; & Print Timer 1 value \\
M02 0; & Stop image processing \\
V1 = V1 + 1; & Increase the cavity counter \\
IF V1 = 8 THEN FINISH; & Judgment \\
G00 X1.5 F1; & Move the electrode to the next position to be \\
GOTO LTESTA; & machined \\
\hline
\end{tabular}

volume (V) have been respectively indicated in Fig. 9. In summary, for the first line the averaged machining time is $1.159 \mathrm{~s}$, the averaged removal volume $0.008699 \mathrm{~mm}^{3}$ and the averaged machining efficiency $0.007506 \mathrm{~mm}^{3} / \mathrm{s}$. For the second line (except for $\# 16$, because there is a large deviation due to some instabilities), the averaged machining time is $2.148 \mathrm{~s}$, the averaged removal volume $0.01651 \mathrm{~mm}^{3}$ and the averaged machining efficiency $0.007686 \mathrm{~mm}^{3} / \mathrm{s}$.

The cavity roundness and the mean absolute error (MAE) of the cavity diameter have been summarized in Fig. 10. The roundness calculation method refers to ref [35], which is similar with minimum zone circle (MZC) method. It can be noticed that the cavities on the second line has better roundness.

The calculation of the cavity diameter and the removal volume is

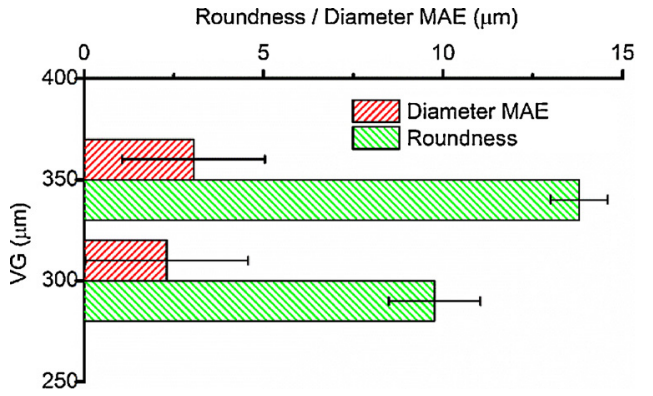

Fig. 10. Roundness and diameter MAE.

based on a free and open source software: Gwyddion. The calculation process is illustrated in Fig. 11. Firstly, the original data from the SENSOFAR surface profiler are imported into Gwyddion. After leveling by three points (Fig. 11(b)), the cavity profile can be figured out by a certain threshold (Fig. 11(c)), which is determined by the height. Finally, the equivalent diameter and the removal volume of the mask region (read region) are calculated through "measure individual grains", which is a built-in function of Gwyddion.

\subsection{Symmetry and stability of the electrolyte droplet}

The rotational symmetry of the electrolyte droplet around the electrode during machining state is evaluated by measuring the movement of the two endpoints of the measured diameter. As show in Fig. 12, if $\left|\Delta L_{1}-\Delta L_{2}\right|<\xi$, the symmetry is acceptable, where $\Delta L_{1}$ and $\Delta L_{2}$ are the moved distance over a certain interval of time, $\xi$ being an artificially selected threshold.

Two main issues lead to the asymmetry of the electrolyte droplet. One is the initial electrode establishment. The other one is due a process blockage caused by the reaction products after a very long time of usage, which hinders the electrolyte recycling.

The stability of the electrolyte droplet is evaluated based on the movement of the diameter endpoints and the standard deviation of the machining current $(\sigma)$. When the machining current is too large or the

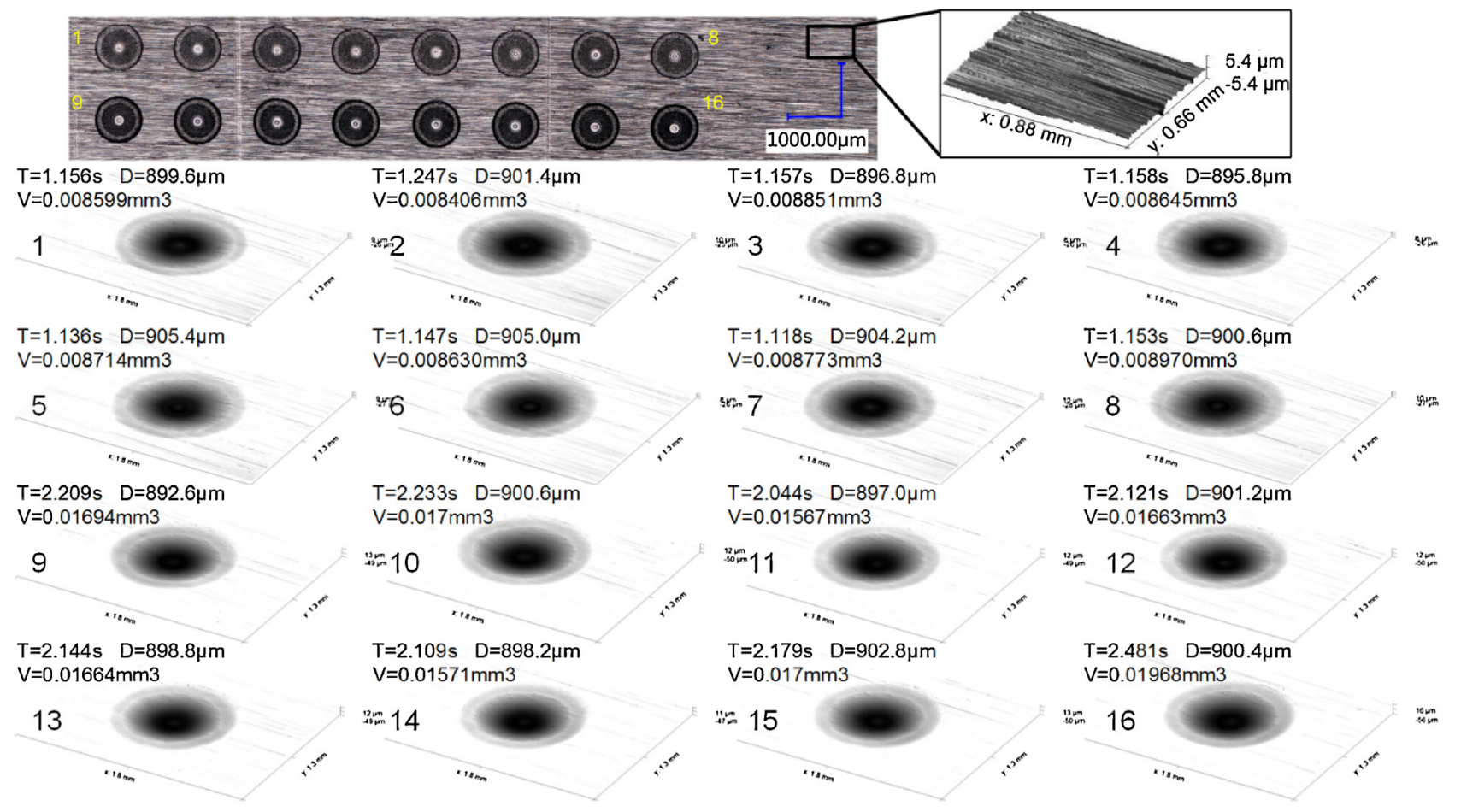

Fig. 9. Cavities machined by the machine vision assisted SMEFC. 


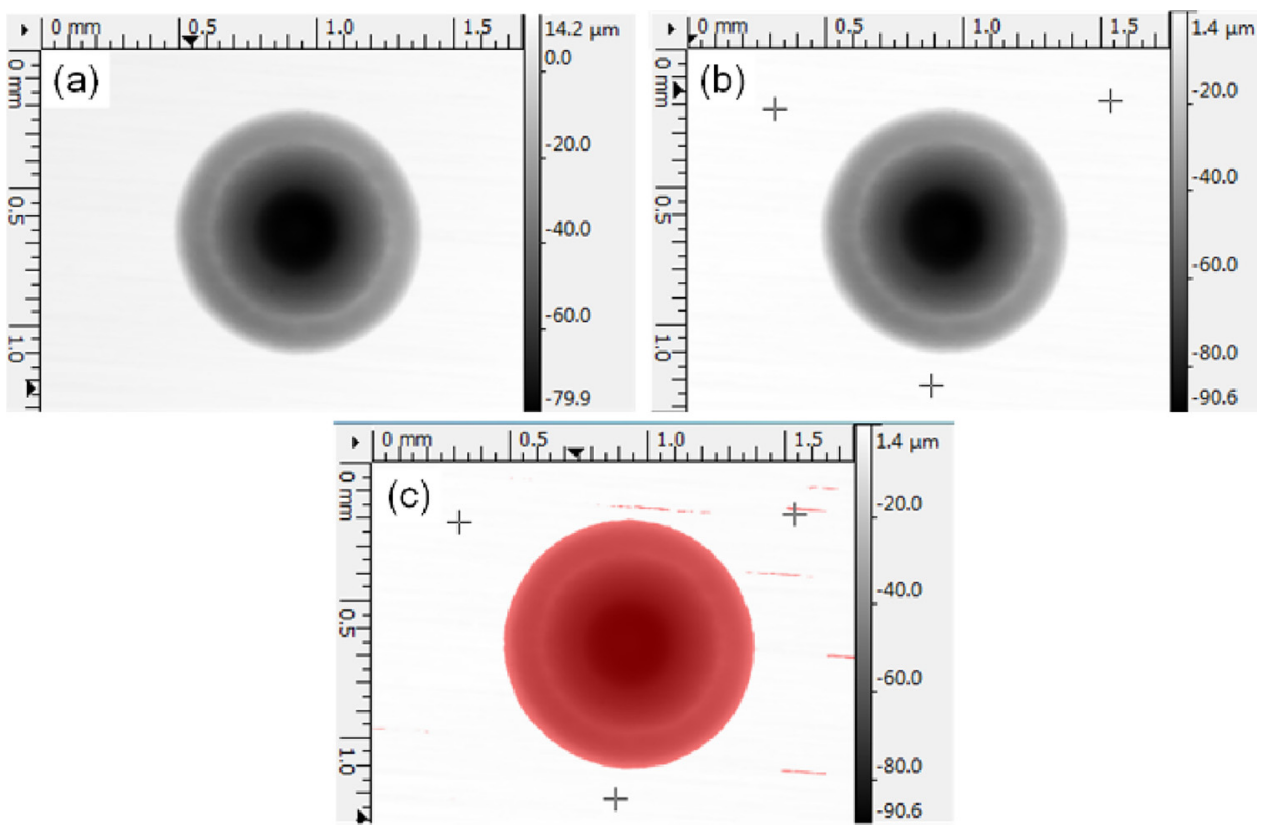

Fig. 11. Diameter and removal volume calculation (a) original data (b) leveling by three points (c) threshold by height.

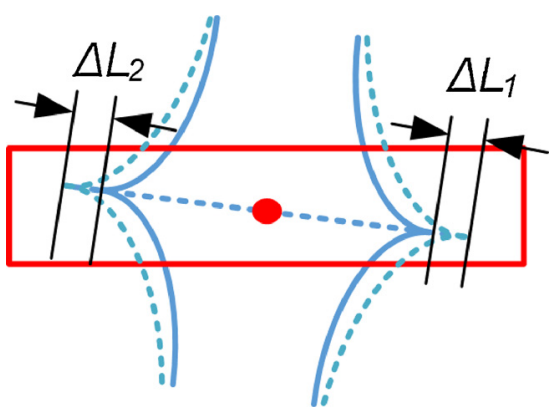

Fig. 12. Schematic of symmetry and stability.

electrolyte flow rate is too low, a large amount of gas bubbles will form due to the electrolyte boiling or strong hydrogen generation. These bubbles tend to change the electrolyte resistance abruptly and even induce discharge, which should be avoided. This kind of problem can be detected by a large $\sigma$ and an abnormal change in the electrolyte droplet morphology. In principle, the standard deviation of the machining current is more sensitive and changing faster than the change in the droplet morphology. The $\sigma$ value is directly calculated in the self-developed ECM power supply and is transferred into the PC. The machining current sampling frequency of the machining current is $200 \mathrm{~Hz}$ and the standard deviation calculation frequency is $10 \mathrm{~Hz}$ as it is based

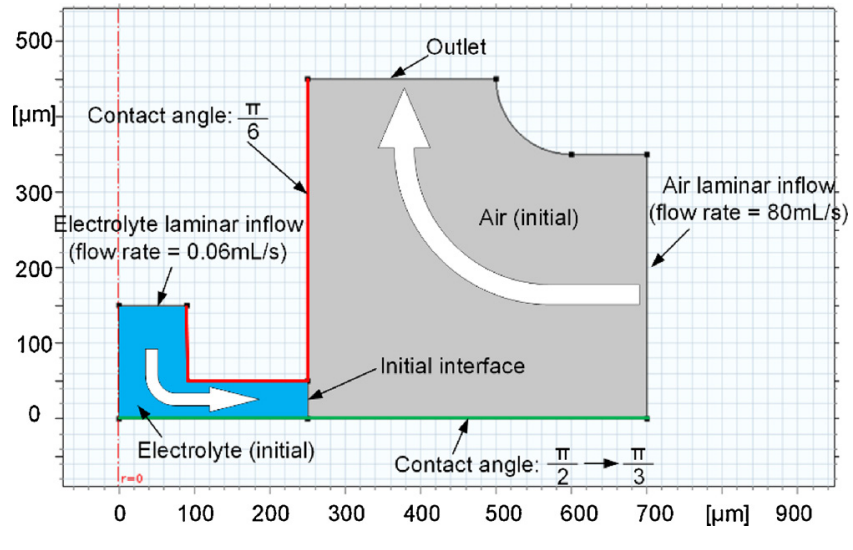

Fig. 14. Geometry of two-phase flow.

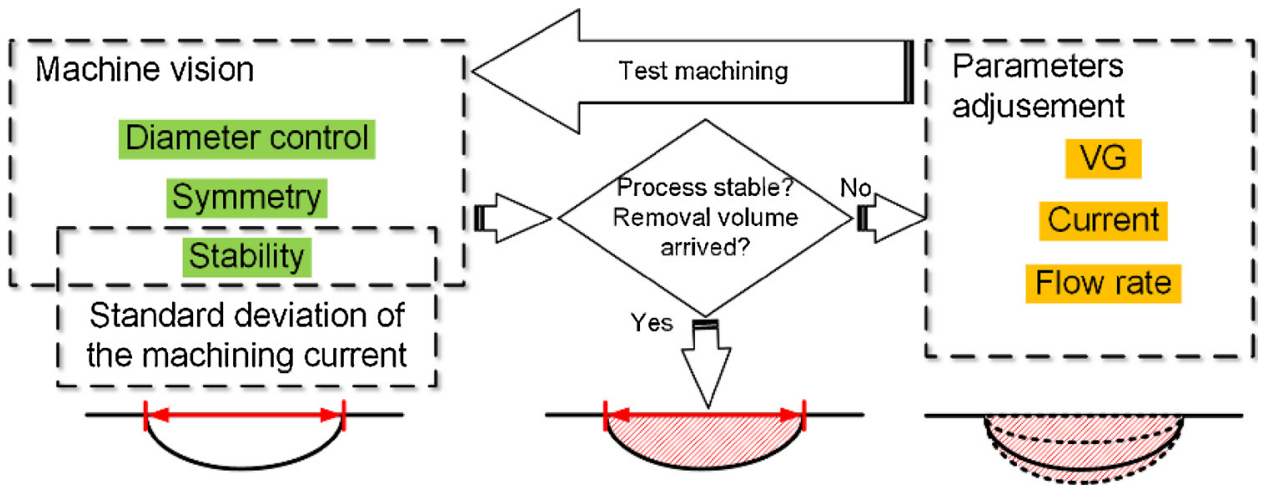

Fig. 13. Deterministic removal strategy. 


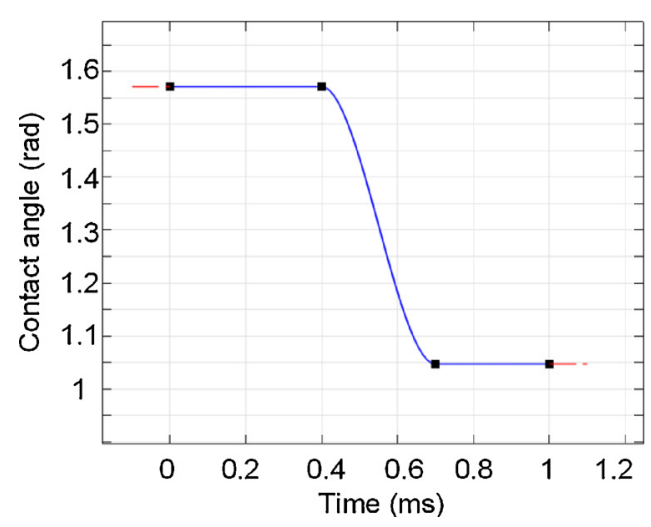

Fig. 15. Assumed contact angle changing process.

Table 4

Properties of the laminar two-phase flow.

\begin{tabular}{lll}
\hline Property & Electrolyte & Air \\
\hline Density & $1142 \mathrm{~kg} / \mathrm{m}^{3}$ & $1.2 \mathrm{~kg} / \mathrm{m}^{3}$ \\
Viscosity & $1.2 \mathrm{~m} \mathrm{~Pa} \mathrm{~s}$ & $0.018 \mathrm{~m} \mathrm{~Pa} \mathrm{~s}$ \\
Surface tension & $0.0725 \mathrm{~N} / \mathrm{m}$ & \\
\hline
\end{tabular}

on 20 current values. In summary, if $\Delta L_{1}<\tau, \Delta L_{2}<\tau$ and $\sigma<\delta$, the stability is acceptable, where $\tau$ and $\delta$ are arbitrarily selected values.

\section{Proposal for a deterministic removal strategy}

\subsection{Parameter tuning strategy}

A detailed deterministic removal strategy is illustrated in Fig. 13. The machine vision is mainly responsible for the diameter control. The adjustable parameters are the VG, the machining current and the electrolyte flow rate. To achieve high material removal rate, a larger machining current may be preferable. A larger current density for the case of $\mathrm{NaNO}_{3}$ electrolyte and stainless steel is also helpful for the surface roughness improvement. The selection of the machining current depends on the Joule heat, gas evolution and limiting current. In principle, the electrolyte flow rate is also capable of altering the machining localization. In practice, the VG is selected as the main control parameter because tuning the VG can provide a more predictable behavior, which will be explained in the following part. Through several rounds of parameter tuning and test machining, suitable parameters can be obtained for the deterministic removal.

\subsection{Theoretical reason for $V G$ tuning}

The adjustment of the VG results in a change of the diameter-depth ratio of the cavity, which has been pointed out in the previous work [16]. However, the reasons behind it were still not clear. In order to explain this phenomenon, a two-phase flow model has been set up using the Level Set (LS) method. Similarly, Hackert-Oschätzchen et al. [36] have successfully applied this method in their study of the dynamic generation of the jet shape in jet-ECM. With the help of this two-phase flow model, not only the phenomenon of electrolyte expansion can be evaluated, but also its physical nature can be investigated.

\subsubsection{Meniscus generation}

When the voltage over the machining gap is zero, a balanced electrolyte droplet can be observed between the electrode and the workpiece, as illustrated in Fig. 3 (b). When a voltage is applied between the electrode and the workpiece, a fast expansion of the electrolyte droplet is observed which is due to and electrowetting phenomenon (Fig. 3 (c)).

To explain this, the electrolyte and the surrounding air are modelled as an axisymmetric two-phase flow model. The geometrical dimensions and boundary conditions are illustrated in Fig. 14. The geometry of this model was established according to the real physical dimensions. The inter-electrode gap (IEG) was set to $50 \mu \mathrm{m}$ and the VG was set to $350 \mu \mathrm{m}$.

Next, the level set interface is to be set up for describing the equations for the convection of the interface. The fluid interface is represented by 0.5 contour of the level set function $\Phi$. In the air $\Phi=1$ and in the electrolyte $\Phi=0$. The level set function can be regarded as the volume fraction of air. The transport of the fluid interface separating the two phases is expressed as

$\frac{\partial \Phi}{\partial \mathrm{t}}+\mathbf{u} \cdot \nabla \Phi=\gamma \nabla \cdot\left(\varepsilon \nabla \Phi-\Phi(1-\Phi) \frac{\nabla \Phi}{|\nabla \Phi|}\right)$

where $\varepsilon$ determines the thickness of the interface, $\gamma$ the amount of reinitialization and $\mathbf{u}$ the velocity. The density and the viscosity are calculated in the following way:

$\rho=\rho_{\text {elec }}+\left(\rho_{\text {air }}-\rho_{\text {elec }}\right) \Phi$

$\mu=\mu_{\text {elec }}+\left(\mu_{\text {air }}-\mu_{\text {elec }}\right) \Phi$

which can guarantee the smoothness of density and viscosity across the fluid interface.

In order to take capillary effects into account, it is also crucial to include surface tension in the equation. The laminar and incompressible Navier-Stokes equations are then written as:

$\rho \frac{\partial \mathbf{u}}{\partial \mathrm{t}}+\rho(\mathbf{u} \cdot \nabla) \mathbf{u}=\nabla \cdot\left(-\mathrm{p} \mathbf{I}+\mu\left(\nabla \mathbf{u}+(\nabla \mathbf{u})^{\mathrm{T}}\right)\right)+\mathbf{F}_{\mathrm{s}}+\rho \mathbf{g}$

$\nabla \cdot \mathbf{u}=0$

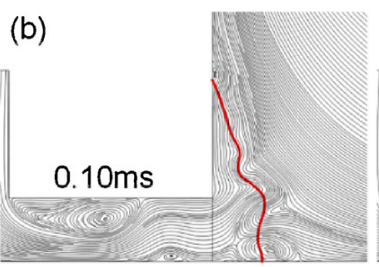

(f)

(e)
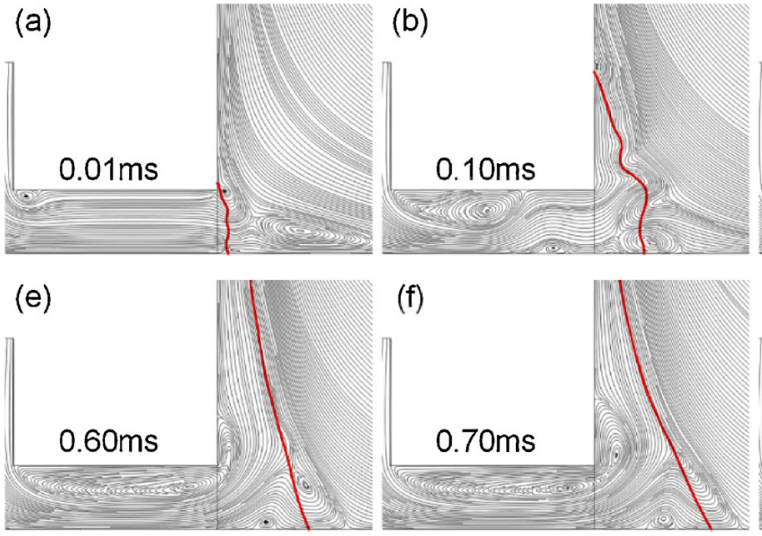

(c)

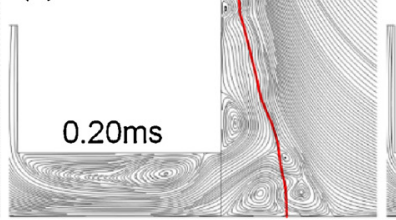

(g)

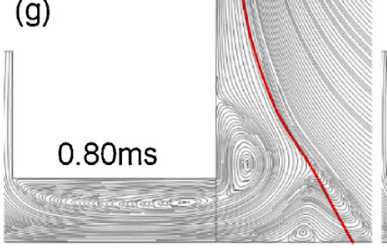

(d)

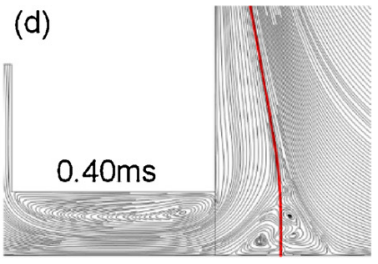

(h)

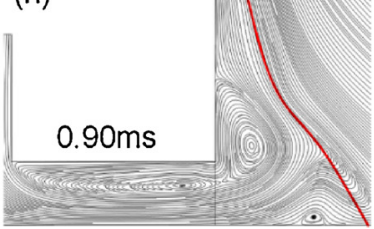

Fig. 16. Streamline and interface evolution (a) $0.01 \mathrm{~ms}$ (b) $0.10 \mathrm{~ms}$ (c) $0.20 \mathrm{~ms}$ (d) $0.40 \mathrm{~ms}$ (e) $0.60 \mathrm{~ms}$ (f) $0.70 \mathrm{~ms}$ (g) $0.80 \mathrm{~ms}$ (h) $0.90 \mathrm{~ms}$. 
(a)

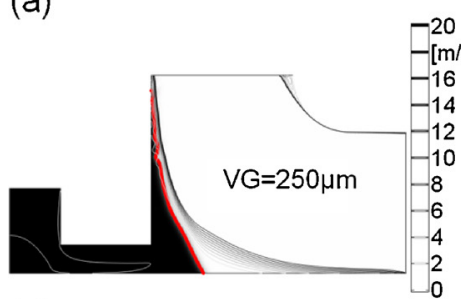

(d)

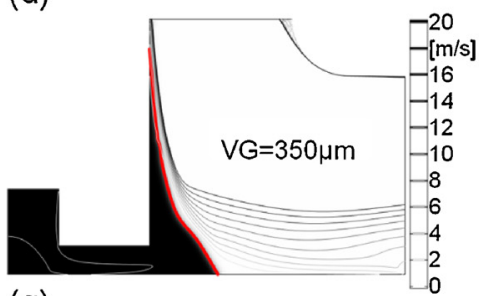

(g) (b)

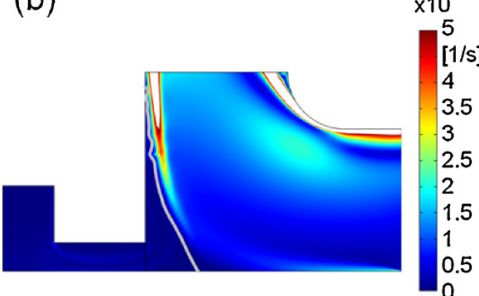

(e)

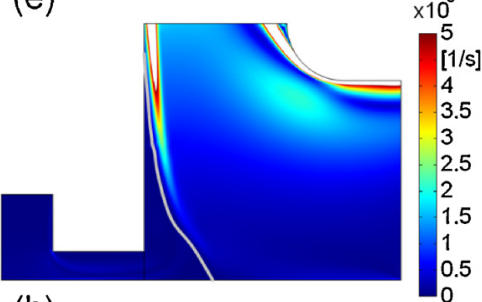

(h)

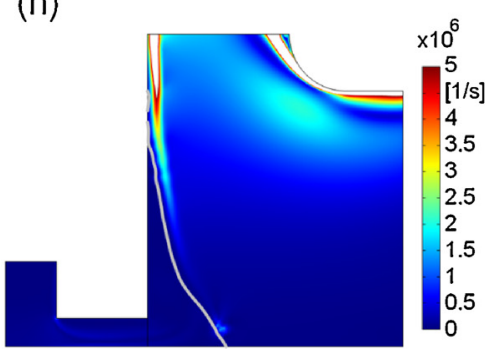

(c)

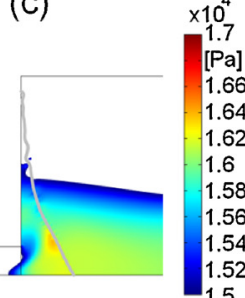

(f)

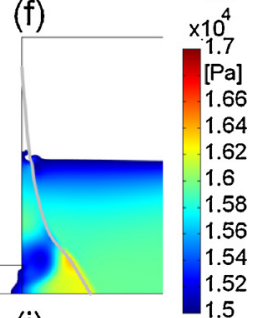

(i)

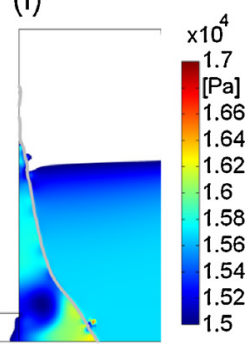

(a)

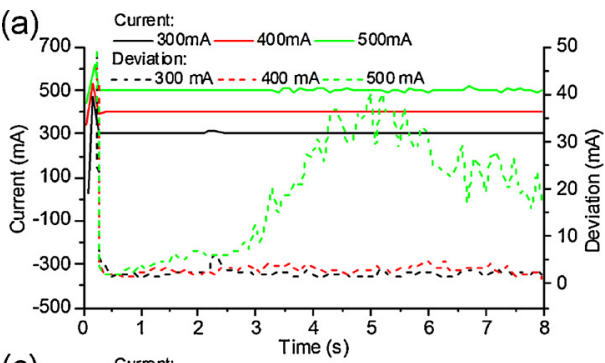

(c)
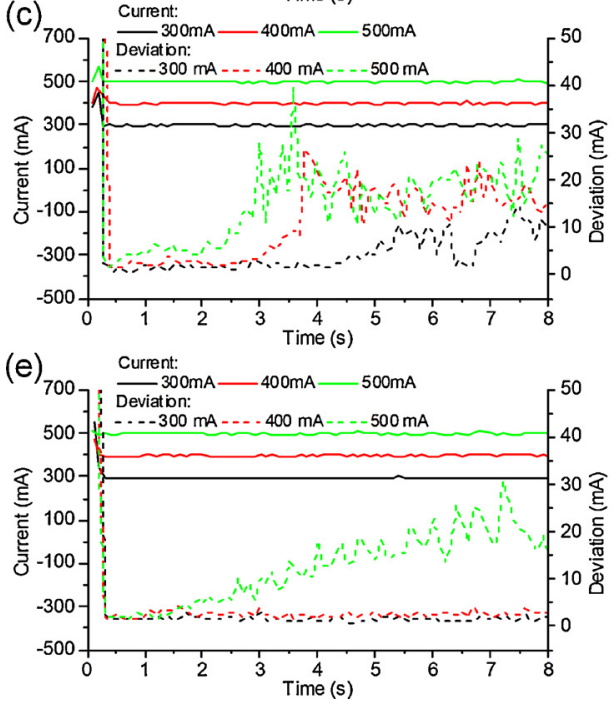

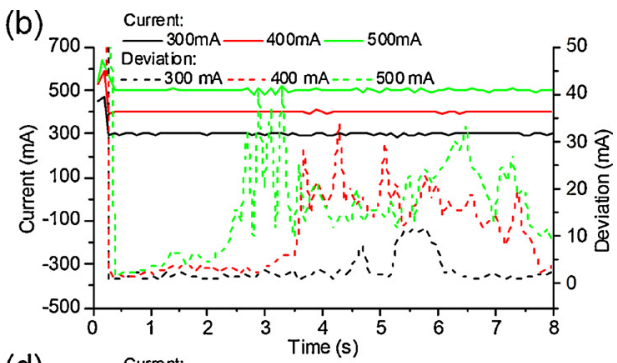

(d)
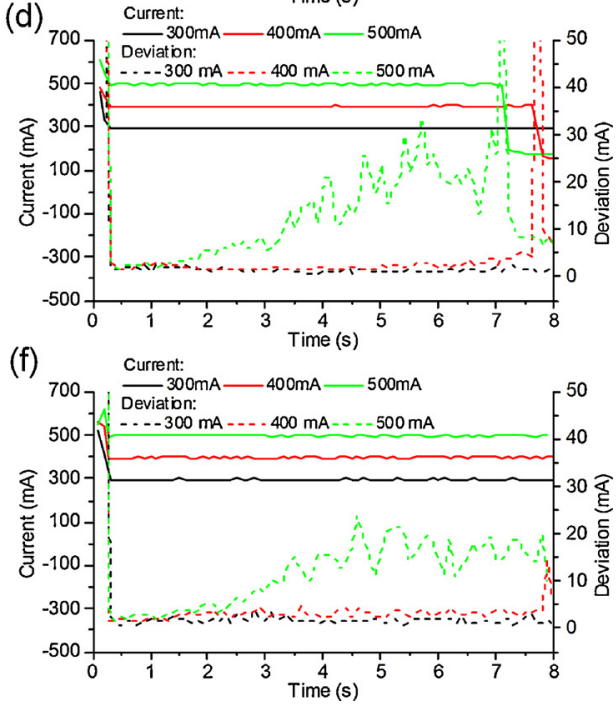

Fig. 17. Velocity contour, shear rate and pressure distribution under different VGs at $\mathrm{t}=0.9 \mathrm{~ms}$ (a) velocity contour and interface $\mathrm{VG}=250 \mu \mathrm{m}$ (b) shear rate VG $=250 \mu \mathrm{m}$ (c) pressure $V G=250 \mu \mathrm{m}$ (d) velocity contour and interface $V G=350 \mu \mathrm{m}$ (e) shear rate $\mathrm{VG}=350 \mu \mathrm{m}$ (f) pressure $\mathrm{VG}=350 \mu \mathrm{m}$ (g) velocity contour and interface $V G=450 \mu \mathrm{m}$ (h) shear rate $\mathrm{VG}=450 \mu \mathrm{m}$ (i) pressure VG $=450 \mu \mathrm{m}$
Fig. 18. Machining current and the its standard deviation (a) $\mathrm{VG}=300 \mu \mathrm{m}$, Flow rate $=3.186 \mathrm{~mL} / \mathrm{min}$ (b) $\mathrm{VG}=400 \mu \mathrm{m}$, Flow rate $=3.186 \mathrm{~mL} / \mathrm{min}$ (c) $\mathrm{VG}=500 \mu \mathrm{m}$, Flow rate $=3.186 \mathrm{~mL} / \mathrm{min}$ (d) $\mathrm{VG}=300 \mu \mathrm{m}$, Flow rate $=2.526 \mathrm{~mL} / \mathrm{min}$ (e) $\mathrm{VG}=400 \mu \mathrm{m}$, Flow rate $=2.526 \mathrm{~mL} / \mathrm{min}(\mathrm{f}) \mathrm{VG}=500 \mu \mathrm{m}$, Flow rate $=2.526 \mathrm{~mL} / \mathrm{min}$. where $\rho$ denotes the density $\left(\mathrm{kg} / \mathrm{m}^{3}\right), \mu$ the dynamic viscosity $\left(\mathrm{N} \cdot \mathrm{s} / \mathrm{m}^{2}\right)$, $\mathrm{p}$ the pressure $(\mathrm{Pa})$, and $\mathbf{g}$ the gravity vector $\left(\mathrm{m} / \mathrm{s}^{2}\right) . \mathbf{F}_{\mathrm{s}}$ is the surface tension force acting at the air/electrolyte interface, which is computed as

$\mathrm{F}_{\mathrm{s}}=\nabla \cdot \mathbf{T}$
$\mathbf{T}=\sigma\left(\mathbf{I}-\left(\mathbf{n n}^{\mathrm{T}}\right)\right) \delta$

where $\mathbf{I}$ is the identity matrix, $\mathbf{n}$ the interface normal, $\sigma$ equals the surface tension coefficient $(\mathrm{N} / \mathrm{m})$, and $\delta$ equals a Dirac delta function that is nonzero only at the fluid interface.

The tool electrode surface and the workpiece surface should be 


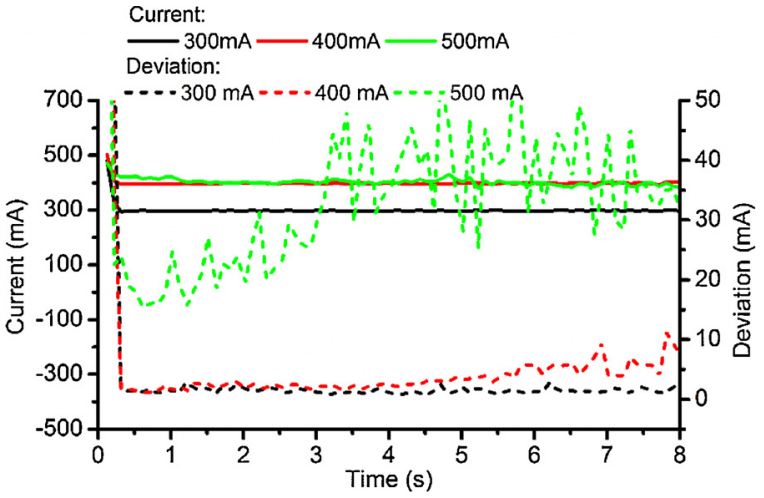

Fig. 19. Machining current and the its standard deviation VG $=300 \mu \mathrm{m}$ Flow rate $=1.752 \mathrm{~mL} / \mathrm{min}$.

regarded as wetted wall boundary condition.

$\sigma\left(\mathbf{n}_{\text {wall }}-\mathbf{n} \cdot \cos \theta\right)=\mathbf{F}_{\text {friction }}=-\frac{\mu}{\beta} \mathbf{u}$

Here, $\beta$ is the slip length, and $\theta$ the contact angle between the wall and the fluid interface.

It is assumed that the contact angle of the electrode surface is $\pi / 6$. The workpiece contact angle with and without voltage is $\pi / 2$ and $\pi / 3$ respectively. It is assumed that the changing of this contact angle happens within $1 \mathrm{~ms}$, as shown in Fig. 15.

The density, viscosity and surface tension values used in this simulation are listed in Table 4.

The simulation procedure consists of two steps. First, the level set functions are initialized, then the time-calculation starts. Fig. 16 shows the streamline of the flow field and the electrolyte/air interface derived from the two-phase flow simulation. The initial development of the fluid interface is shown in Fig. 16 (a), (b), (c) and (d). During this stage the surface changes drastically in order to maintain the prescribed contact angle with the workpiece surface. When the contact angle $(\pi / 2)$ is achieved, the electrolyte is lifted along the electrode side surface due to the surface tension and the surrounding fast moving air.

When a voltage is applied over the IEG, the contact angle of the workpiece changes from $\pi / 2$ to $\pi / 3$ according to the assumptions made. Fig. 16 (e), (f), (g) and (h) shows the evolution process. Compared with the original state, the new state showed a larger wetted area and meniscus, which is the result of the interactions of the electrolyte and the surrounding air.

Fig. 16 also clearly demonstrates how the vortexes evolve in the electrolyte domain. As shown in Fig. 16 (h), three visible vortexes form, which tend to influence the electrochemical dissolution of the workpiece. In the air domain, the vortex near the workpiece surface which was present during the initial stage (Fig. 16 (a)) disappears in Fig. 16 (h).

\subsubsection{Effects of the $V G$}

To investigate the influence of the VG, the two-phase flow simulation has been conducted for three levels of the VG. Fig. 17 illustrates the velocity contour $(0 \sim 20 \mathrm{~m} / \mathrm{s})$, interface (red line), shear rate and pressure distribution. In Fig. 17 (a), (d) and (g), it can be noticed that with the increase of the VG the contact area also increases. The air velocity gradient along the meniscus on the other hand decreases, which contributes to the reduction in the shear rate near the interface (Fig. 17 (b), (e) and (h)). On the contrary, the pressure near the workpiece surface (Fig. 17 (c), (f) and (i)) is larger than the pressure around the upper part. From the point of view of this simulation, the shear effect and the pressure difference both force the electrolyte to rise along the electrode surface. The difference in the shear effect and the pressure difference for different values of the VG can theoretically explain how the VG influences the contact area. In addition, a pressure jump of roughly tens of $\mathrm{Pa}$ across the interface can be observed due to the surface tension.

In summary, the VG setting effectively influences the shear rate and pressure difference near the meniscus and further influences the wetted area. Therefore, the VG is selected as the main tuning parameter, since it provides a more predictable behavior of the droplet geometry.

\subsection{Selection of the other process parameters}

The machining current influences the gas generation and heat generation. Flowing electrolyte can flush away gas bubbles and heat. In order to select both a suitable machining current and electrolyte flow rate, the standard deviation of the machining current is taken as an evaluation index. The constant-current mode is utilized. The initial voltage is set to $25 \mathrm{~V}$. With the increase of the VG, an increase of the standard deviation of the machining current is observed as shown in Fig. 18 (a), (b) and (c). It is also demonstrated that at a higher machining current, the standard deviation is always higher, which can be
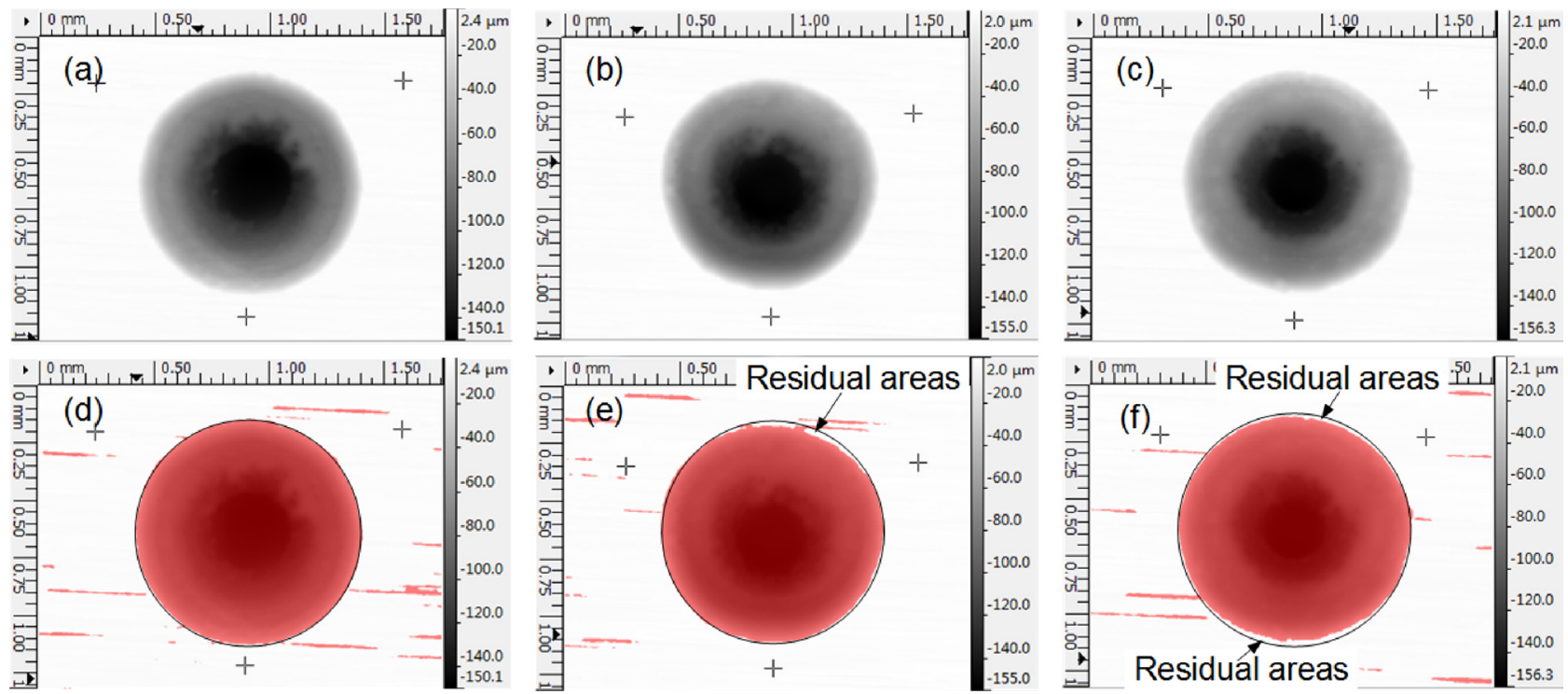

Fig. 20. Cavities processed with the current of $300 \mathrm{~mA}$ and the VG of $400 \mu \mathrm{m}$ without and with read masks: (a), (d) Flow rate $=3.186 \mathrm{~mL} / \mathrm{min}$ (b), (e) Flow rate $=2.526 \mathrm{~mL} / \mathrm{min}$ (c), (f) Flow rate $=1.752 \mathrm{~mL} / \mathrm{min}$. 


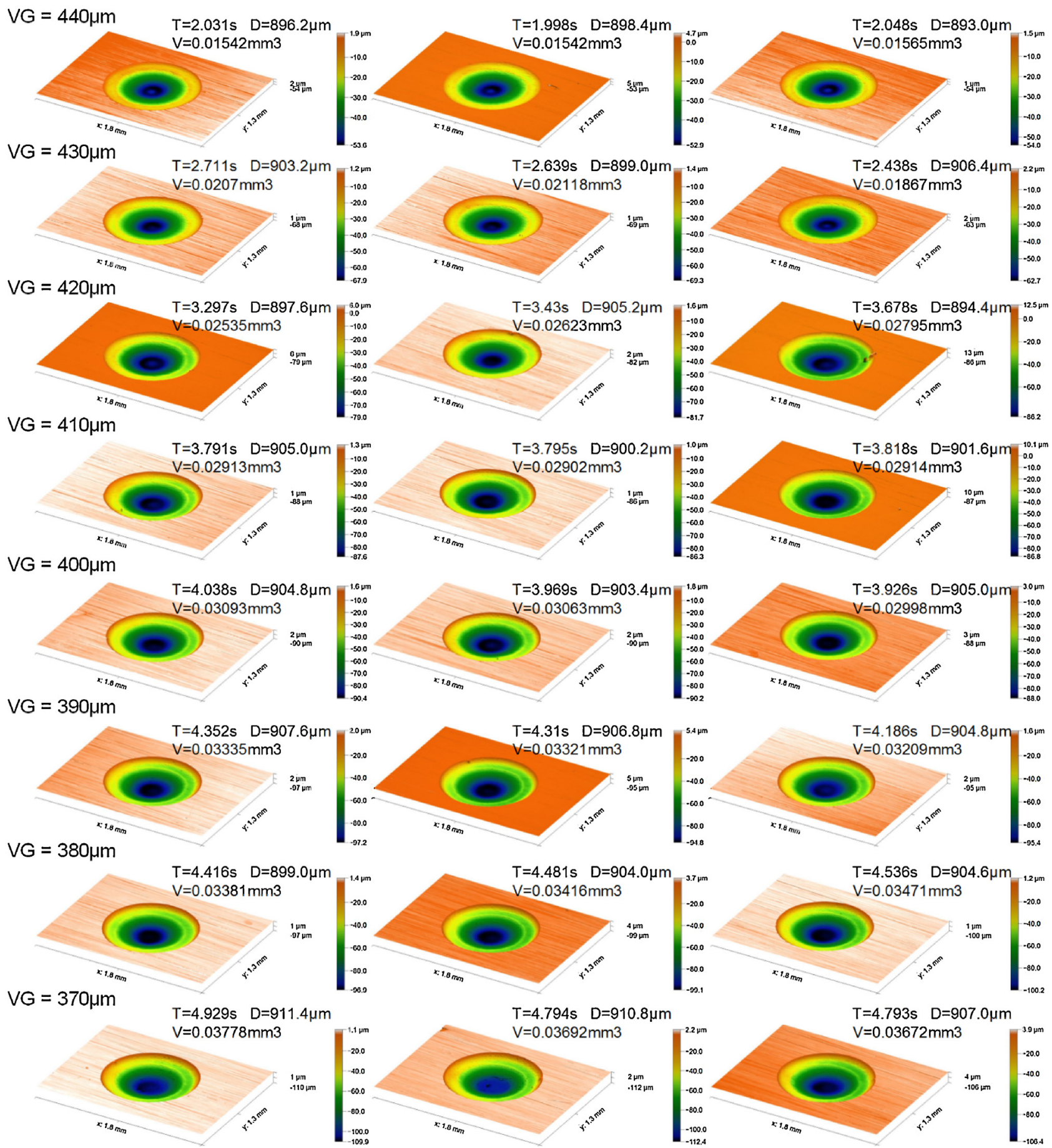

Fig. 21. Cavity topography and measurement data under different VGs.

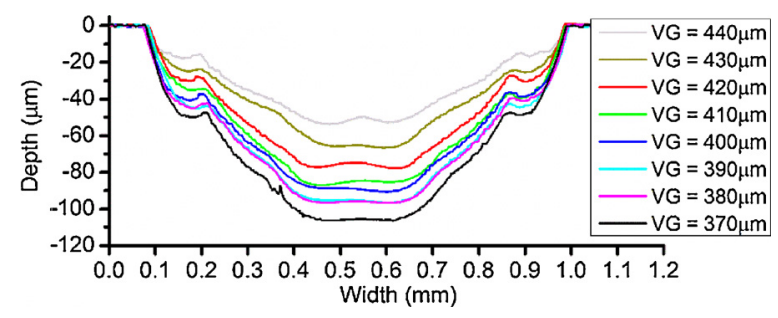

Fig. 22. Cross-sectional profiles of the cavities.

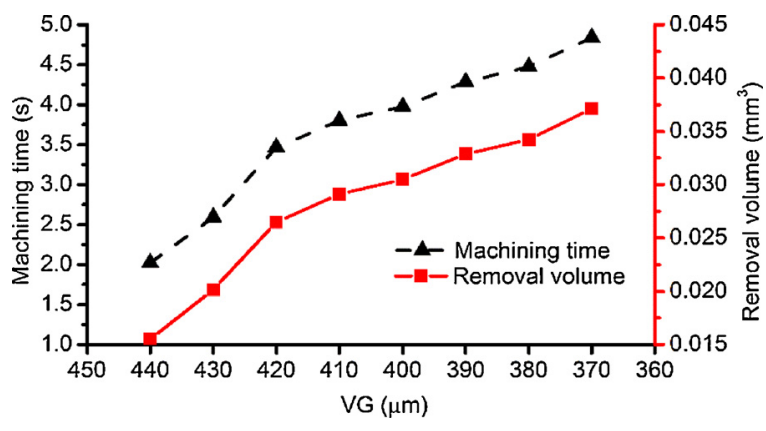

Fig. 23. Averaged machining time and removal volume. 


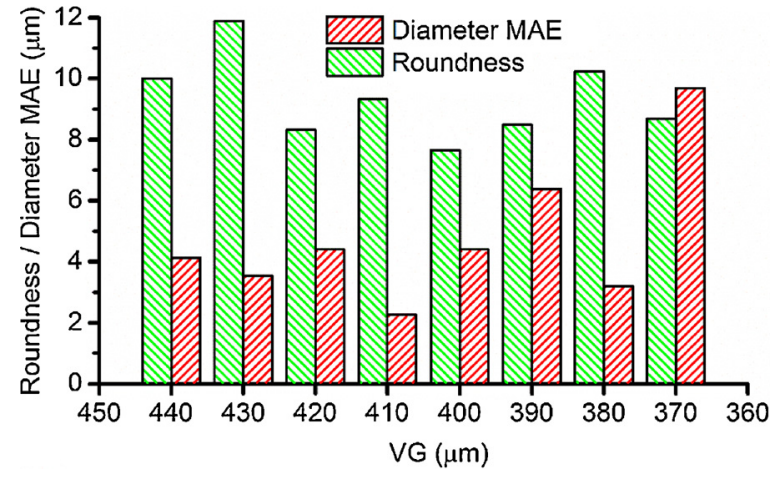

Fig. 24. Roundness and diameter MAE.

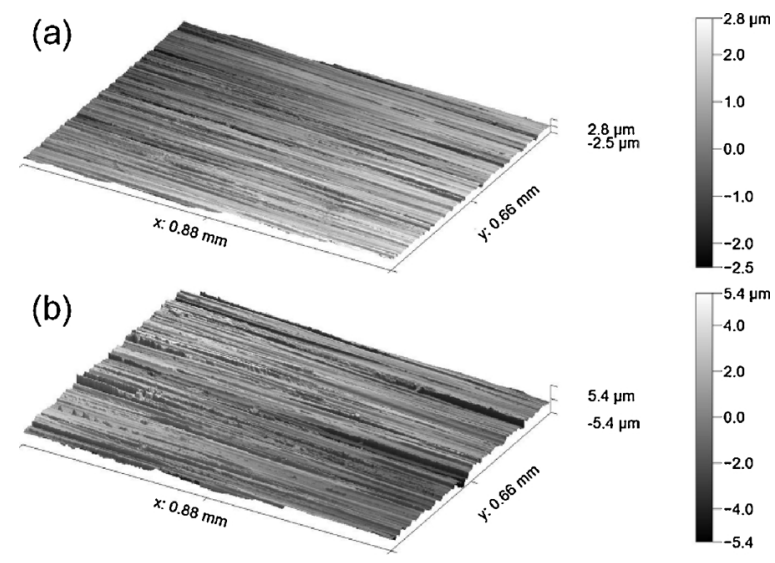

Fig. 25. Surface morphology (a) Original surface in Section 4 (b) Original surface in Section 2.3 .

attributed to the intensive generation of bubbles. For the cases with a flow rate of $3.186 \mathrm{~mL} / \mathrm{min}$, an increase in the standard deviation happens at $2.5 \mathrm{~s}$ for $500 \mathrm{~mA}$ and $3.5 \mathrm{~s}$ for $400 \mathrm{~mA}$. For the machining current of $300 \mathrm{~mA}$, the maximum standard deviation is around $10 \mathrm{~mA}$. It can be noticed that when the flow rate is set to $2.526 \mathrm{~mL} / \mathrm{min}$, the standard deviation is smaller than that where the flow rate was $3.186 \mathrm{~mL} / \mathrm{min}$, especially for the first $7 \mathrm{~s}$.

Fig. 19 shows the standard deviation for the flow rate of $1.752 \mathrm{~mL} /$ min. It can be found that $500 \mathrm{~mA}$ cannot be achieved although the maximum voltage is applied. The possible reason is that low electrolyte flow rate cannot effectively flush away gas bubbles that are blocking the electrochemical reactions in the gap.

Fig. 20 demonstrates the projected shape of the cavities processed under different flow rates. The red mask on it is to indicate the cavity. Compared to the case with $3.186 \mathrm{~mL} / \mathrm{min}$ (Fig. 20 (d)), lower flow rates (Fig. 20 (e) and (f)) induce a larger difference from the standard circle, although a lower flow rate leads to a smaller standard deviation of the machining current, as shown in Fig. 18. Furthermore, it can be deduced that machining at the flow rate of $2.562 \mathrm{~mL} / \mathrm{min}$ and $1.752 \mathrm{~mL} / \mathrm{min}$ tends to be affected by the original texture on the workpiece to be machined. For example, when looking at the residual areas within the standard circles of Fig. 20 (e) and (f), it can be found that the residual areas inside the standard circle always appear at the upper and lower region, which means material removal is easier to happen along the grinding mark direction. The red marks outside the standard circle are the grinding marks. Considering both the machining current stability and the cavity shape deviation, the values of $300 \mathrm{~mA}$ machining current and $3.186 \mathrm{~mL} / \mathrm{min}$ flow rate are finally selected.

\section{Case study}

A case study is used to illustrate the use of the developed technology. The envisaged cavity has a target diameter of $900 \mu \mathrm{m}$ and the targeted removal volume is $0.03 \mathrm{~mm}^{3}$. In order to avoid the errors induced by the re-clamping, a VG ranging from $440 \mu \mathrm{m}$ to $370 \mu \mathrm{m}$ with intervals of $10 \mu \mathrm{m}$ was tested using a machining current of $300 \mathrm{~mA}$ and an electrolyte flow rate of $3.186 \mathrm{~mL} / \mathrm{min}$. Three tests were conducted for each VG. The cavity topography and measurement data are illustrated in Fig. 21. With the decrease of the VG, the maximum depth of the cavity increases from around $50 \mu \mathrm{m}(\mathrm{VG}=440 \mu \mathrm{m})$ to around $110 \mu \mathrm{m}(\mathrm{VG}=370 \mu \mathrm{m})$. Fig. 22 shows the cross-sectional profiles of the cavities in the left column of Fig. 21.

Fig. 23 shows the averaged machining time and removal volume under 8 levels of VG. When the VG is set to $400 \mu \mathrm{m}$, the averaged removal volume is $0.03051 \mathrm{~mm}^{3}$ and the averaged machining time is $3.977 \mathrm{~s}$. In this way, the required machining time and the VG value can be obtained for deterministic removal. Fig. 24 indicates the roundness and the diameter MAE of the cavities. It can be noticed that the averaged roundness is $9.33 \mu \mathrm{m}$. The averaged diameter MAE is $4.75 \mu \mathrm{m}$.

From this case study, it can be concluded that for SMEFC the suitable machining parameters can be quickly found to achieve a deterministic removal with the help of the proposed machine vision assisted system. Another interesting phenomenon is that the machining time for the cavity with the diameter of $900 \mu \mathrm{m}$ in Section 2.3 is $1.159 \mathrm{~s}$ (VG $=350 \mu \mathrm{m})$, smaller than all the machining times mentioned on Fig. 21, although smaller VGs have been applied in Section 2.3. This can be explained using the Wenzel's model that describes the contact angle on a rough surface [37].

$\cos \theta^{*}=r \cos \theta$

where $\theta^{*}$ is the apparent contact angle under the minimum free energy state, $r$ the ratio of the true area of the solid surface to the apparent area (roughness ratio) and $\theta$ the Young contact angle for an ideal surface.

Fig. 25 demonstrated the 3D profiles of both workpiece surfaces. The ground surface in Fig. 25 (a) is smoother than that of Fig. 25 (b). The peak-to-peak value of the former is around $5 \mu \mathrm{m}$ and that of latter is $10 \mu \mathrm{m}$. Apparently, the workpiece surface roughness ratio of Section 4 is smaller than that of Section 2.3, so a smaller apparent contact angle of Section 2.3 can be expected according to the Wenzel's model. The simulation in Section 3.2 has revealed that the wetted area increases with the reduction in the contact angle of the workpiece surface.

From the above discussion, it can be understood that the surface profile influences the electrolyte droplet behavior at the initial moment. In other words, the machining characteristics of SMEFC are quite sensitive to the original surface conditions, although the workpiece material is identical. This property highlights the significance of the proposed machine vision assisted system, because the system allows to control the machining characteristics of SMEFC for a certain surface condition.

\section{Conclusion}

- Since small cavities have a positive influence on the sliding performance, the aim of this work is to explore a fast way to quickly acquire suitable parameters, by which small cavities with a specified diameter and a specified removal volume can be fabricated by electrochemical dissolution. In this context, a deterministic removal strategy for machine vision assisted scanning micro electrochemical flow cell (SMEFC) machining has been proposed.

- This machine vision assisted system uses the visual information of the electrolyte droplet during the machining process to realize an on-line control of the cavity diameter. The core algorithm of this system is the Shi-Tomasi corner detector. The applicability of this system has been verified by generating a set of 8 cavities with a target diameter of $900 \mu \mathrm{m}$. 
- The vacuum gap (VG) has been selected as the main parameter to adjust the removal volume. Through a two-phase model of SMEFC, it is concluded that the formation mechanism of the electrolyte droplet is based on a combination of the shear effect and the pressure difference near the meniscus. Furthermore, decreasing the VG can strengthen the shear effect, increase the pressure difference, and finally reduce the wetted area, which explains why the VG setting can enhance the machining localization.

- A case study has finally demonstrated the feasibility of the proposed deterministic removal strategy. The introduction of this machine vision assisted system enables to push scanning micro electrochemical flow cell (SMEFC) machining to a more applicable level.

\section{Acknowledgments}

The authors would like to thank the China Scholarship Council (CSC) for providing a scholarship. The authors also would like to thank Mr. Vivian Chang at AnMo Electronics Corp. for the suggestions on the driver for the Dino-lite microscope and Mr. Liang Fang at TME, KU Leuven for the fruitful discussions on the two-phase flow model. The authors appreciate the assistance from Mr. Shashwat Kushwaha, Mr. Xingzhi Xiao, Mr. Krishna Kumar Saxena and Mr. Mattia Bellotti at PMA, KU Leuven during the preparations for the experiments and characterization of the workpiece.

\section{References}

[1] Etsion I. State of the art in laser surface texturing. J Tribol 2005;127:248-53.

[2] Andersson P, Koskinen J, Varjus Se, Gerbig Y, Haefke H, Georgiou S, et al. Microlubrication effect by laser-textured steel surfaces. Wear 2007;262:369-79.

[3] Brizmer V, Kligerman Y, Etsion I. A laser surface textured parallel thrust bearing. Tribol Trans 2003;46:397-403.

[4] Yan D, Qu N, Li H, Wang X. Significance of dimple parameters on the friction of sliding surfaces investigated by orthogonal experiments. Tribol Trans 2010;53:703-12.

[5] Siripuram RB, Stephens LS. Effect of deterministic asperity geometry on hydrodynamic lubrication. J Tribol 2004;126:527-34.

[6] Teixidor Ezpeleta D, Grzenda M, Bustillo Iglesias A, Ciurana Qd. Modeling pulsed laser micromachining of micro geometries using machine-learning techniques, (C). J Intell Manuf 2015;26(4):801-14. 2015.

[7] Zhu D, Qu N, Li H, Zeng Y, Li D, Qian S. Electrochemical micromachining of microstructures of micro hole and dimple array. CIRP Ann-Manuf Technol 2009;58:177-80.

[8] Hao X, Wang L, Wang Q, Guo F, Tang Y, Ding Y, et al. Surface micro-texturing of metallic cylindrical surface with proximity rolling-exposure lithography and electrochemical micromachining. Appl Surf Sci 2011;257:8906-11.

[9] Schubert A, Neugebauer R, Sylla D, Avila M, Hackert M. Manufacturing of surface microstructures for improved tribological efficiency of powertrain components and forming tools. CIRP J Manuf Sci Technol 2011;4:200-7.

[10] Walker J, Kamps T, Lam J, Mitchell-Smith J, Clare AT. Tribological behaviour of an electrochemical jet machined textured Al-Si automotive cylinder liner material. Wear 2017;376:1611-21.

[11] Natsu W, Ikeda T, Kunieda M. Generating complicated surface with electrolyte jet machining. Prec Eng 2007;31:33-9.

[12] Hackert-Oschätzchen M, Meichsner G, Zinecker M, Martin A, Schubert A. Micro machining with continuous electrolytic free jet. Prec Eng 2012;36:612-9.

[13] Kawanaka T, Kunieda M. Mirror-like finishing by electrolyte jet machining. CIRP
Ann-Manuf Technol 2015;64:237-40

[14] Chen X, Qu N, Li H, Guo Z. Removal of islands from micro-dimple arrays prepared by through-mask electrochemical micromachining. Prec Eng 2015;39:204-11.

[15] Wang G, Li H, Qu N, Zhu D. Improvement of electrolyte flow field during throughmask electrochemical machining by changing mask wall angle. J Manuf Process 2017;25:246-52.

[16] Guo C, Qian J, Reynaerts D. electrochemical machining with scanning micro electrochemical flow cell (SMEFC). J Mater Process Technol 2017;247:171-83.

[17] Guo C, Qian J, Reynaerts D. Fabrication of mesoscale channel by scanning micro electrochemical flow cell (SMEFC). Micromachines 2017;8:143.

[18] Yan M-T, Huang K-Y, Lo C-Y. A study on electrode wear sensing and compensation in micro-EDM using machine vision system. Int J Adv Manuf Technol 2009;42:1065-73.

[19] Fernández-Robles L, Azzopardi G, Alegre E, Petkov N. Machine-vision-based identification of broken inserts in edge profile milling heads. Robot Comput-Integr Manuf 2017;44:276-83.

[20] Wang Z, Liu R, Sparks T, Liu H, Liou F. Stereo vision based hybrid manufacturing process for precision metal parts. Prec Eng 2015;42:1-5.

[21] Liu R, Wang Z, Sparks T, Liou F, Nedic C. Stereo vision-based repair of metallic components. Rapid Prototyp J 2017;23:65-73.

[22] Abdul-Ameer HK, Al-Kindi GA, Zughaer H. Towards computer vision feedback for enhanced cnc machining, in: communication software and networks (ICCSN). 2011 IEEE 3rd International Conference on, IEEE. 2011. p. 754-60.

[23] Sitthi-Amorn P, Ramos JE, Wangy Y, Kwan J, Lan J, Wang W, et al. MultiFab: a machine vision assisted platform for multi-material 3D printing. ACM Trans Graph 2015;34:129.

[24] Supriadi S, Manabe K. Enhancement of dimensional accuracy of dieless tubedrawing process with vision-based fuzzy control. J Mater Process Technol 2013;213:905-12.

[25] Huang C, Tarng Y, Chiu C, Huang A. Investigation of machine vision assisted automatic resharpening process of micro-drills. J Mater Process Technol 2009;209:5944-54.

[26] Lertrusdachakul I, Mathieu A, Aubreton O. Vision-based control of wire extension in GMA welding. Int J Adv Manuf Technol 2015;78:1201-10.

[27] Zhu J, Wang J, Su N, Xu G, Yang M. An infrared visual sensing detection approach for swing arc narrow gap weld deviation. J Mater Process Technol 2017;243:258-68.

[28] Liu X, Jia C, Wu C, Zhang G, Gao J. Measurement of the keyhole entrance and topside weld Pool geometries in keyhole plasma arc welding with dual CCD cameras. J Mater Process Technol 2017;248:39-48.

[29] Klocke F, Zeis M, Herrig T, Harst S, Klink A. Optical in situ measurements and interdisciplinary modeling of the electrochemical sinking process of inconel 718. Procedia CIRP 2014;24:114-9.

[30] Shimasaki T, Kunieda M. Study on influences of bubbles on ECM gap phenomena using transparent electrode. CIRP Ann-Manuf Technol 2016;65:225-8.

[31] Rebschläger A, Kollmannsperger R, Bähre D. Video based process observations of the pulse electrochemical machining process at high current densities and small gaps. Procedia CIRP 2014;14:418-23.

[32] Paczkowski T, Zdrojewski J. Monitoring and control of the electrochemical machining process under the conditions of a vibrating tool electrode. J Mater Proces Technol 2017;244:204-14.

[33] Chong ZZ, Tor SB, Gañán-Calvo AM, Chong ZJ, Loh NH, Nguyen N-T, et al Automated droplet measurement (ADM): an enhanced video processing software for rapid droplet measurements. Microfluid Nanofluid 2016;20:66.

[34] Shi J. Good features to track, in: computer vision and pattern recognition. Proceedings CVPR'94., 1994 IEEE Computer Society Conference on, IEEE, 1994. 1994. p. 593-600.

[35] Guo C, Saxena KK, Bellotti M, Qian J, Reynaerts D. Fast mesoscale texturing by multi-electrode scanning micro electrochemical flow cell. Procedia CIRP 2018;68:741-5.

[36] Hackert-Oschätzchen M, Paul R, Martin A, Meichsner G, Lehnert N, Schubert A. Study on the dynamic generation of the jet shape in jet electrochemical machining. J Mater Process Technol 2015;223:240-51.

[37] Marmur A. Wetting on hydrophobic rough surfaces: to be heterogeneous or not to be? Langmuir 2003;19:8343-8. 